\title{
Design of a Low-Cost Air Quality Monitoring System Using Arduino and ThingSpeak
}

\author{
Anabi Hilary Kelechi ${ }^{1}$, Mohammed H. Alsharif ${ }^{2}$, Chidumebi Agbaetuo ${ }^{3}$, Osichinaka Ubadike ${ }^{1}$ \\ Alex Aligbe ${ }^{1}$, Peerapong Uthansakul ${ }^{4, *}$, Raju Kannadasan ${ }^{5}$ and Ayman A. Aly ${ }^{6}$
}

\footnotetext{
${ }^{1}$ Department of Aerospace Engineering, College of Engineering, AirForce Institute of Technology, Kaduna, Nigeria ${ }^{2}$ Department of Electrical Engineering, College of Electronics and Information Engineering, Sejong University, Seoul, 05006, Korea

${ }^{3}$ Department of Electrical Engineering and Information Engineering, College of Engineering, Covenant University, Canaanland, Ota, P.M.B 1023, Ogun, Nigeria

${ }^{4}$ School of Telecommunication Engineering, Suranaree University of Technology, Nakhon Ratchasima, Thailand ${ }^{5}$ Department of Electrical and Electronics Engineering, Sri Venkateswara College of Engineering, Chennai, 602117, India ${ }^{6}$ Department of Mechanical Engineering, College of Engineering, Taif University, Taif, 21944, Saudi Arabia *Corresponding Author: Peerapong Uthansakul. Email: uthansakul@sut.ac.th
} Received: 13 April 2021; Accepted: 14 May 2021

\begin{abstract}
The impact of daily emissions of gaseous and particulate pollutants of machines and industries on human health and the environment has attracted increasing concerns. This impact has significantly led to a notable increase in mortality in the highly industrialized zones. Therefore, monitoring air quality and creating public awareness are important for a safer future, which led the governments globally to invest multi-billion in policymaking and solution stratification to address the problem. This study aims to design a realtime Internet of Things low-cost air quality monitoring system. The system utilizes air quality and carbon monoxide sensors for monitoring gaseous pollutants. Moreover, the system utilizes an Arduino Nano development board equipped with a WiFi module to effectively send readings to a ThingSpeak online channel platform for instantaneous and real-time display of air quality. The ThingSpeak uses HTTP protocols to send emails in raising awareness of poor air quality. The level of concentration is monitored graphically through channels with the help of ThingSpeak to aid remote communication. A threshold value is set. Thus, when pollutants have become unhealthy and harmful, the system trips off an alarm, and e-mail notifications are sent to the officials. The results have shown that the work was successfully implemented a design of a low-cost air quality monitoring system using Arduino and ThingSpeak, showing that an air quality system can be implemented using a low-cost technology, Arduino and ThingSpeak.
\end{abstract}

Keywords: IoT; air quality; MQ 135 sensor; MQ-7 sensor; air pollution 


\section{Introduction}

Maintaining adequate air quality possesses a global challenge to governments and citizens. The deteriorating air quality has essentially caused governments globally to invest in multi-billion sums in policymaking and solution stratification to address the problem. Air pollution is caused by particulate matter emitted from industries, cars, machinery, waste recycling, industrial practices, and household. Some of the notable pollutants are the dust of heavy metals, carbon monoxide, ozone, carbon dioxide, nitrogen dioxide, suspended particulate matter, hydrogen fluoride oxides of sulfur, and others. These pollutants get into the atmosphere and cause severe health and environmental effects. In a survey conducted in 2016, the World Health Organization (WHO) stated that air pollution, specifically of the ambient (outdoor) origin, is the estimated cause of 4.2 million premature deaths annually worldwide [1]. This value is a reflection of the population living in rural and urban areas. The map in Fig. 1 shows the density and intensity of air pollution globally.

Average Annual Population-Weighted PM2.5 Concentrations in 2017

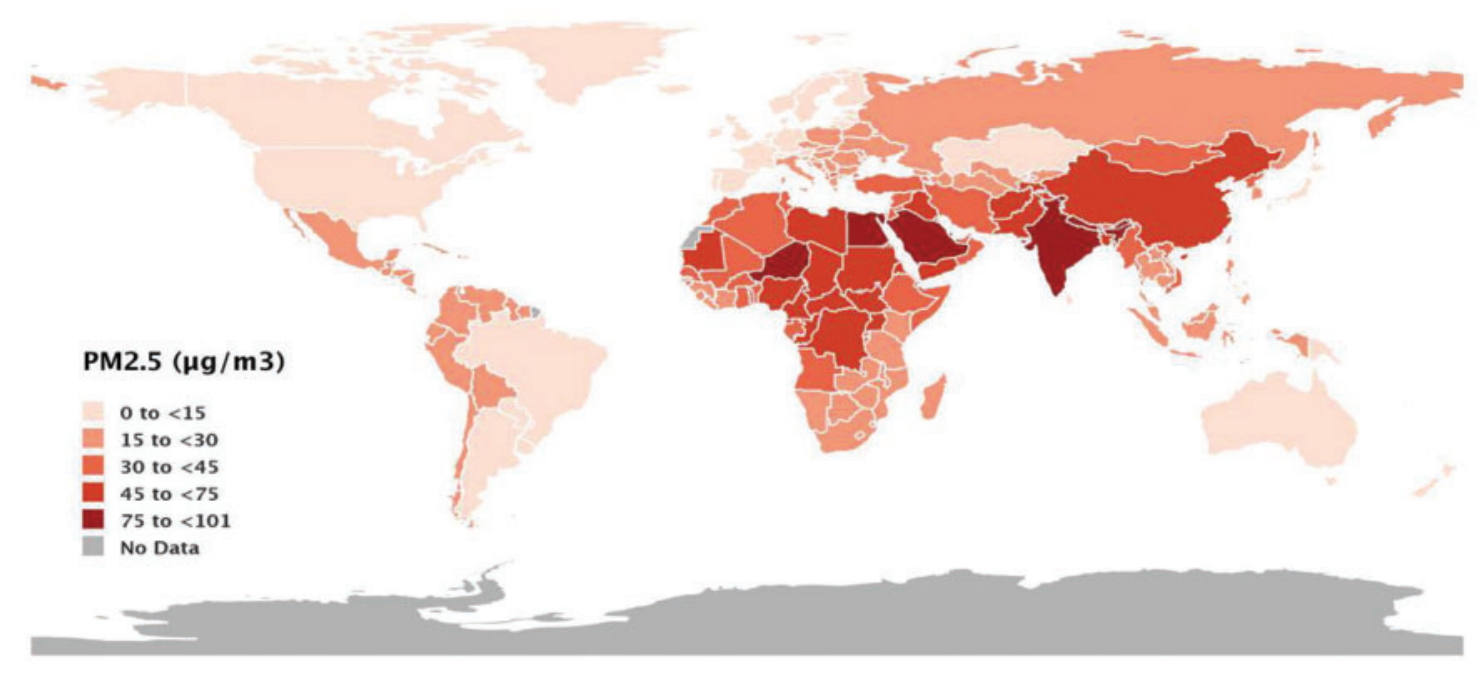

State of Global Air

Figure 1: PM 2.5 concentrations around the world [1]

Air pollution impacts our lives and generations yet born; hence, we should be cautious and control harmful emissions of particulate matter into the atmosphere. A step in achieving this goal is by designing a cheap real-time air quality monitoring system. Internet of Things (IoT) is a game-changing innovation in the technology, policy, industry, and engineering circles that can enable air quality monitoring [2]. IoT allows the connection of a network of objects by incorporating intelligent sensing systems, such as radio-frequency identification, sensors, and two-dimensional codes into a "physical entity," to obtain information about the purpose at any time [3]. IoT connects a range of devices through the Internet to communicate and exchange data and utilize such interconnection to conduct highly beneficial functions.

Borghi et al. [4] noted that the significant problems faced by the traditional air monitoring system are the relatively sophisticated hardware innovation, unsafe operation, high cost, and 
bulky instruments (Fig. 2). Furthermore, for the equipment to provide accurate precision and performance, sophisticated statistical methods aided with devices, such as sensors, filters, humidity, and temperature monitors, are necessary. However, these methods require an excessive amount of energy from the massive and expensive equipment [5]. Hence, they are not energy-efficient and sustainable. The conventional techniques do not make the system efficient for remote monitoring and cause inadequacy in detecting the rise in pollution concentration [6]. Ideally, sensors that are efficient, cheap, and of relative size are the best choice for monitoring the ambient air. These devices are employed in industries but are partly used in environmental monitoring due to the inefficiency to measure massive data of specific gas pollutants under ambient cases. Hence, lowcost sensors will have to be analyzed using the traditional monitoring equipment to compare their efficiency [7]. To date, detailed and comprehensively distributed estimates comparing productivity are generally uncommon, particularly for diagnostic advancements, which are now being used [8].

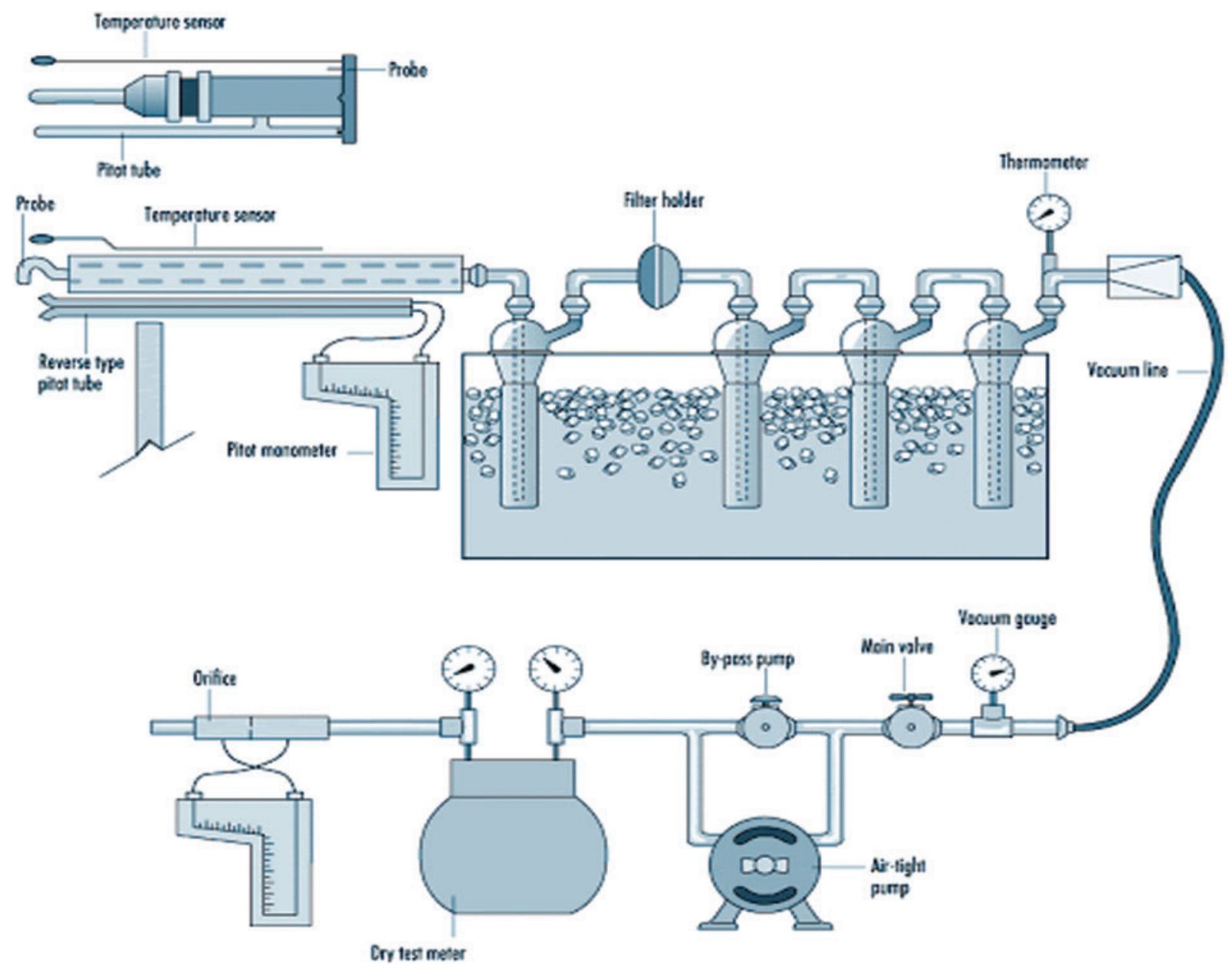

Figure 2: Traditional air quality monitoring device

The study aims to develop a low-cost, convenient, high-precision, portable air pollution monitoring device that is capable of measuring the concentration of various types of particulate matter pollutants in the air under real-time architecture using the IoT platform. The system 
working principle was subdivided into sub-routine/objectives and tested to ensure that the system is efficiently functioning. The goals set out to achieve are the following:

- Design a web server system that measures air quality index (AQI) in the environment.

- Interface the system with a liquid crystal display (LCD) module that displays the AQI of the situation.

- Integrate the system with an IoT platform to enable remote monitoring of the AQI.

This paper is further organized as follows. Section 2 details a standard and structured review of past work in this area of study, drawing parallels between them and highlighting weaknesses and strengths. Section 3 introduces the methodologies used in this system design, explaining the methods and the reasons for using such methods. Then, Section 4 presents the actual design and implementation. Section 5 provides the results and discussions. Finally, Section 6 concludes the study.

\section{Literature Review}

The AQI is a numerical value representation system created by the Environmental Protection Agency (EPA) to make a logical way of reporting daily air quality concerning the environment and human health. The AQI focuses on the negative impacts that people may experience on their health while breathing polluted air over a few hours or days. An increase in the AQI represents a subsequent increase in air pollution and human health threats [9]. The AQI is calculated for the six major pollutants stated under the Clean Air Act by the EPA in 1970: nitrogen oxides, particulate matter (PM 10, PM 2.5), carbon monoxide, sulfur dioxide, and ground-level ozone. Recent advances in technology have led to creating innovations and opportunities, such as the IoT, whereby embedded systems connected to the Internet can interact and be controlled through the Internet. For instance, researchers deployed IoT technology to monitor the water quality of a lake [10]. Numerous research projects on wireless sensory networks or IoT to detect and monitor the AQI has been performed [11]. These concluded projects measured various air pollutants with the use of mobile or stationary sensors.

The proposed system evaluates the Waspmote platform created by Libelium, which offers various radio technologies, sensors, application programming interface (API), and open-source software development kits to improve neural networks [12]. This study evaluates the power consumption of wireless sensor networks, identified analytical functions, and then created a framework with utilization parameters. Reference [13] proposed a system using a wireless monitoring system to measure concentrations of pollutants. The proposed system was implemented on an IoT platform using sensory nodes connected to the Internet via a network gateway. The researchers used sensory nodes to ascertain the concentration of carbon dioxide and carbon monoxide in the environment.

This proposed system employs mobile sensors placed on city buses to symbolize a smart air pollution system named "OpenSense" [14]. In this project, the researchers deployed trams as mobile platforms for conveying sensors. The sensors communicate to the cloud through a radio device cloud platform. Then, [15] proposed a system that monitors outdoor air parameters. The system consists of a PIC18F87K22 microcontroller interface with infrared gas and aerometry sensors. The researchers strategically placed the nodes embedded with the neural network at some locations in the town. This paper proposed the utilization of the IEEE/ISO/IEC 21451 standard to monitor air quality [16]. The system uses infrared and electrochemical sensors to measure $\mathrm{NO}_{2}, \mathrm{CO}, \mathrm{SO}_{2}$, and $\mathrm{CO}_{2}$ concentration. The results were relayed and stored on a data server. 
Then, [17] implemented a system to create awareness of the effects of pollutants, gases, and toxins on air quality. The proposed system analyzes meteorological data, traffic data, and contaminants using an intelligent business engine called "APA." The system detects the trends in air pollution and reports human activities that influence the decline in air quality. Meanwhile, [18] proposed a framework for observing environmental air quality. The system consists of a low-cost raspberry pi module used in establishing the system. The proposed system monitored air pollution parameters, such as $\mathrm{CO}_{2}, \mathrm{CO}$, pressure, and temperature, but did not consider PM, making it incomplete for its function. Reference [19] also proposed a system driven by a neural network that used nodes to measure concentrations. The authors used temperature and humidity sensors to make the system more accurate. From another perspective, [20] examined vehicular GPRS-aided air sensors using a smartphone monitoring platform to measure concentrations of PM 2.5. Moreover, [21] proposed a smartphone monitoring platform and air quality surveillance system using sensory arrays and an IoT mobile platform to enable users to receive concentration readings. Similarly, an environmental monitoring system was used to measure ambient air data by utilizing a smartphone monitoring platform with complex architecture sensor network models and mobile API systems.

\section{Materials and Methods}

This section describes an IoT-based air quality monitoring system comprising the hardware components and the software platforms. Some of the tasks performed include design specification, design concept, and materials and method.

\subsection{Design Specification}

The air quality monitoring system comprises five units as shown in Tab. 1. The first unit is a power supply unit, which consists of a DC jack and a regulator. The second unit is the sensing unit, which consists of an air quality sensor and a carbon monoxide sensor. Then, the third unit is the control unit, which consists of the Arduino Uno atmega microcontroller. The fourth unit is the communication unit, which consists of a WiFi module used to transfer the air quality status from the control unit to the IoT platform. Finally, the fifth unit is the output unit, which consists of an LCD, a buzzer, and a ThinkSpeak IoT platform.

Table 1: Design components

\begin{tabular}{lll}
\hline S/N & Units & Components \\
\hline 1 & Power supply unit & DC Jack, 5 V voltage regulator LM7805, resistors \\
2 & Sensing unit & MQ135 air quality monitor sensor, MQ7 CO sensor \\
3 & Microcontroller unit (MCU) & Arduino Uno Atmega microcontroller \\
4 & Communication unit & ESP8266 WiFi module \\
5 & Output unit & $16 \times 2$ LCD monitor, Buzzer, ThinkSpeak IoT platform \\
\hline
\end{tabular}

\subsection{Design Concept and System Flowchart}

Fig. 3 depicts the proposal's design concept, whereas Fig. 4 shows the flowchart of the system with the description highlighted as follows. 


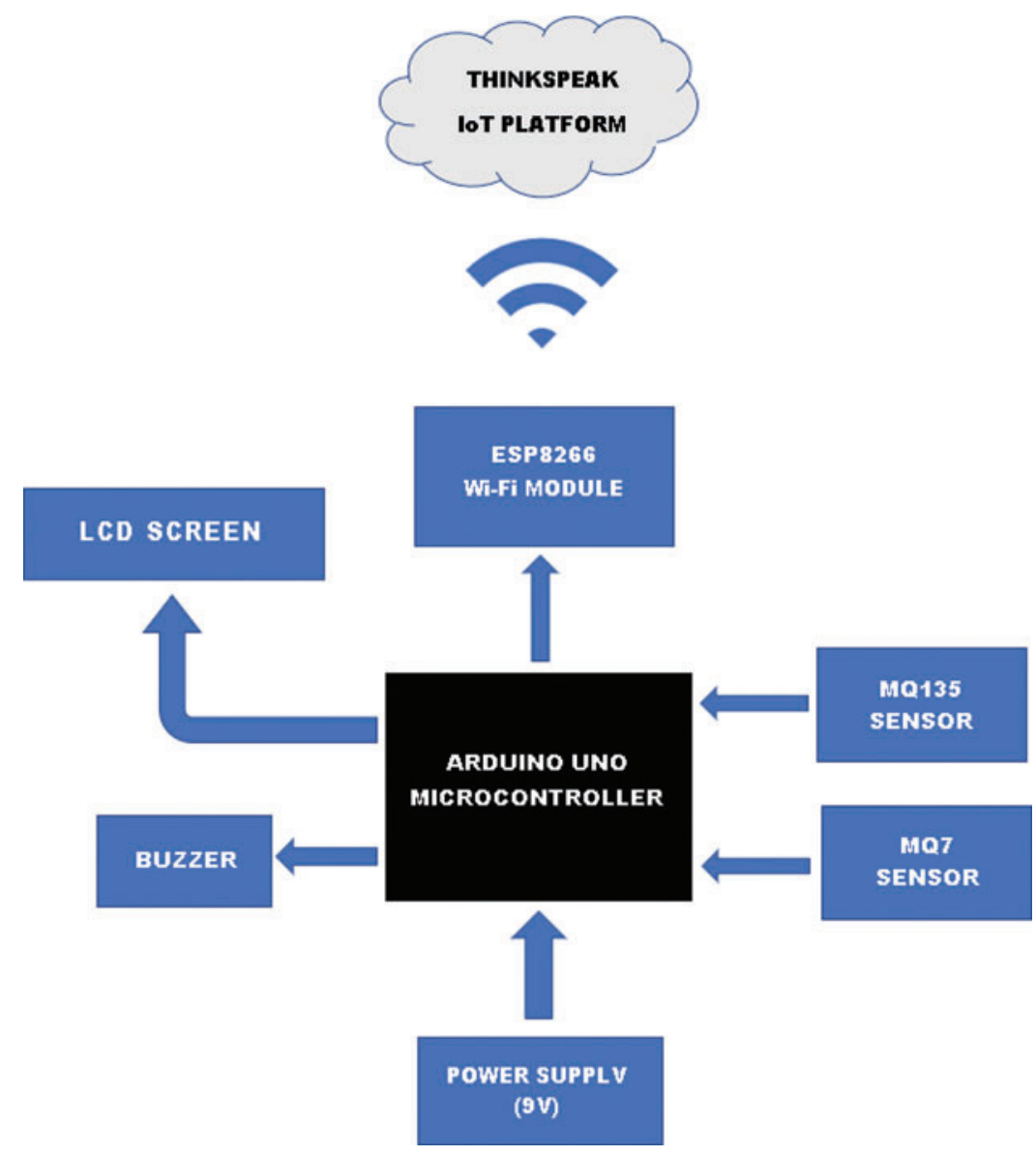

Figure 3: Block diagram of the system

(i) A DC jack supplies power to the system. The DC jack is connected to the MCU, which controls the sub-units.

(ii) An MQ135 air quality sensor is used to measure the concentration of air pollutants in the atmosphere. An MQ-7 sensor is designed for measuring the concentration of carbon monoxide in the atmosphere.

(iii) A programmed Arduino Uno microcontroller is the brain of the system that conducts the needed mathematical operations regarding the comparison between the received analogue signals from the sensors and the preset value. This microcontroller also synchronizes tasks relating to other components in the system.

(iv) An ESP8266 WiFi module is used as the gateway to allow the Arduino Uno microcontroller to interact with the ThinkSpeak IoT platform to write data to its channels.

(v) A $16 \times 2$ LCD monitor is used to display the level of concentration of air pollutants in the atmosphere.

(vi) The Thinkspeak IoT platform shows a virtual representation of the written data in its channel, which also enables the values to be remotely accessed. 


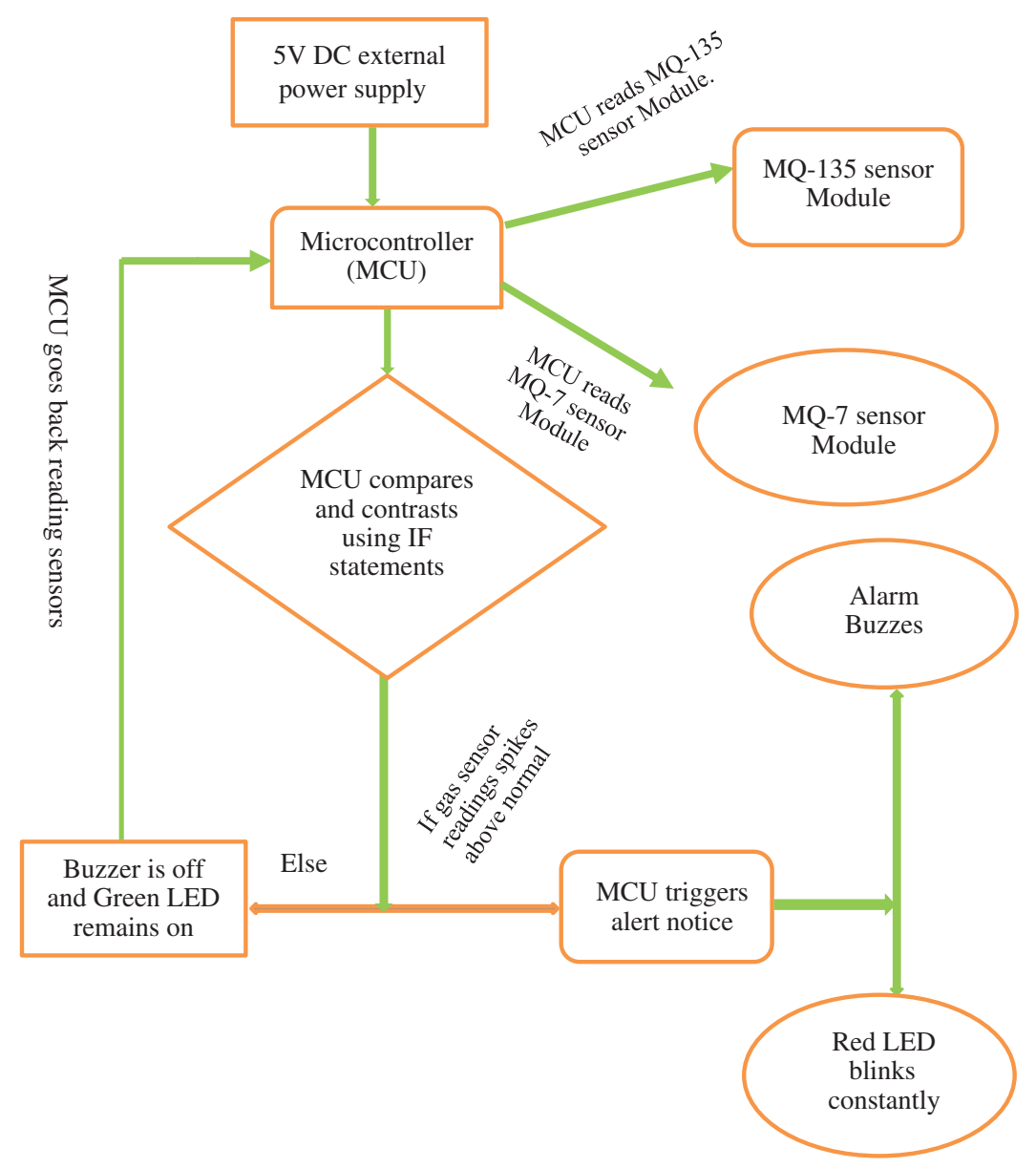

Figure 4: Flowchart of the project

\subsection{Hardware Design}

The air quality monitoring system design is divided into two interdependent sections, that is, the software and the hardware. This section briefly describes the hardware design of the system.

\subsubsection{Choice of the Microcontroller}

The project uses two harmful gas concentration sensors, namely, MQ-135 and MQ-7. These two sensors have a maximum of four IO (input-output) pins. Each is powered by a working mode at constant $5 \mathrm{~V}$. Using analog read protocols is best for the readings of these sensors. Hence, our microcontroller choice was from the Arduino nano-board, which has an on-board Atmega328P IC, 28 pins, and works on $5 \mathrm{~V}$. Tab. 2 presents the summarized microcontroller pin requirement.

\subsubsection{Arduino Nano-Board}

The nano-board offers excellent flexibility and compartment and is also a low-cost development board. Tab. 3 shows the technical specification according to the datasheet. 
Table 2: Microcontroller pin requirement calculations

\begin{tabular}{ll}
\hline Components & Pin requirement \\
\hline MQ135 pins & 2 \\
MQ7 pins & 2 \\
Internet access module (UART) & 3 \\
LED indicators & 2 \\
Buzzer & 1 \\
Total required pins & 10 \\
\hline
\end{tabular}

Table 3: Technical specification of the nano-board

\begin{tabular}{ll}
\hline Components & Specifications \\
\hline Microcontroller & ATmega328P-8-bit AVR family microcontroller \\
Operating voltage & $5 \mathrm{~V}$ \\
Recommended input voltage for Vin & $7-12 \mathrm{~V}$ \\
Analog input pins & 6 (A0-A5) \\
Digital I/O pins & 14 (of which, 6 provide PWM output) \\
DC current on I/O pins & $40 \mathrm{~mA}$ \\
DC current on $3.3 \mathrm{~V}$ pins & $50 \mathrm{~mA}$ \\
Flash memory & $32 \mathrm{~KB}(2 \mathrm{~KB}$ is used for bootloader) \\
SRAM & $2 \mathrm{~KB}$ \\
EEPROM & $1 \mathrm{~KB}$ \\
Frequency (clock speed) & $16 \mathrm{MHz}$ \\
Communication module & $\mathrm{IIC}, \mathrm{SPL}, \mathrm{USART}$ \\
\hline
\end{tabular}

\subsubsection{Air Quality Sensor}

This gas sensor is susceptible to tin oxide compound $(\mathrm{SnO} 2)$, which has lower conductivity in clean air. Fig. 5a illustrates a typical air quality sensor, whereas Fig. 5b depicts the MQ 135 sensor. Thus, the gas sensor works; when the target combustible gas exists within its range of sensory field, the sensor will change its electrical conductivity, which can be used with a microcontroller to map out a range of threshold values. Moreover, the sensor can convert the change of conductivity to correspond output signal of gas concentration. This MQ135 gas sensor module has a high sensitivity to ammonia, sulfide, and benzene steam. Furthermore, the MQ135 gas sensor can be used in sensitive nature to detect $\mathrm{NO}_{2}$.

\subsubsection{Carbon Monoxide Sensor}

The MQ-7 gas sensor measures carbon monoxide and smoke in the atmosphere, as shown in Fig. 6. This sensor has the potential to detect different harmful gases and other concentrations of dangerous combustible gases. The MQ-7 gas sensor can operate at a temperature between -20 and $50^{\circ} \mathrm{C}$. This sensor can consume less than $150 \mathrm{~mA}$ current at $5 \mathrm{~V}$ rating and has the accuracy of detecting gas concentrations between 100 and 10,000 ppm. The module version of this sensor makes it very easy for the connection. Although this sensor is more expensive, the project design was more accurate in taking air quality measurements to determine the module version's toxic gas 
concentration level. Two options may be used: either getting a digital output (DOUT) or analog output (AOUT). The technical specifications are as follows:

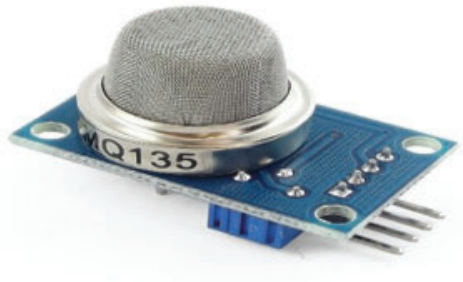

(a)

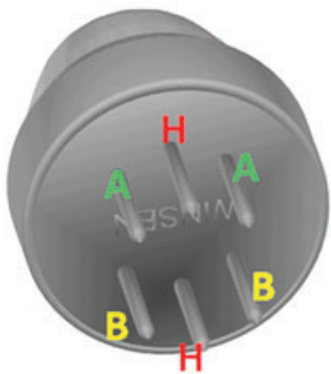

(b)

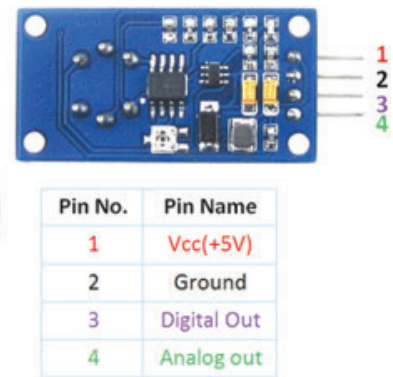

(b)

Figure 5: Typical air quality sensor. (a) Air quality sensor (b) Configuration of the MQ 135 sensor

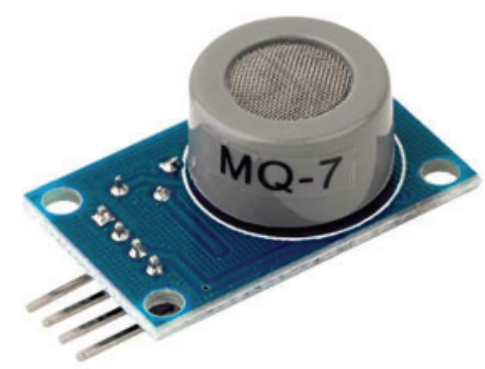

Figure 6: Carbon monoxide sensor

- Operating voltage is $+5 \mathrm{~V}$.

- The sensor can be used to measure or detect LPG, alcohol, propane, hydrogen, CO, and even methane.

- Analog output voltage is from 0 to $5 \mathrm{~V}$.

- Digital output voltage is from 0 or $5 \mathrm{~V}$ (TTL Logic).

- Preheat duration is $20 \mathrm{~s}$.

- The sensor can be used in digital or analog.

- The sensitivity of the digital pin can be varied using a potentiometer.

\subsubsection{ESP8266 WiFi Module}

The ESP8266 ESP-01 is a WiFi module that can allow MCUs to access a WiFi network, as shown in Fig. 7. The module comes with a system on chip configuration, which means that this module does not need a microcontroller to manipulate input and output pins. Thus, the ESP-01 is provided with properties to act like a small computer. The ESP8266 has nine GPIOs (General Purpose Input Output). With the ESP8266 module acting as a module, we can have Internet access once connected to a WiFi network as the module is connected to the MCU in Station (STA) mode. The ESP8266 WiFi module is a module that can connect to the Internet. Thus, in this IoT project, the user has the option to check the level of noise or air quality via web or mobile phones visible on an IoT dashboard. The module has two modes of operation. 


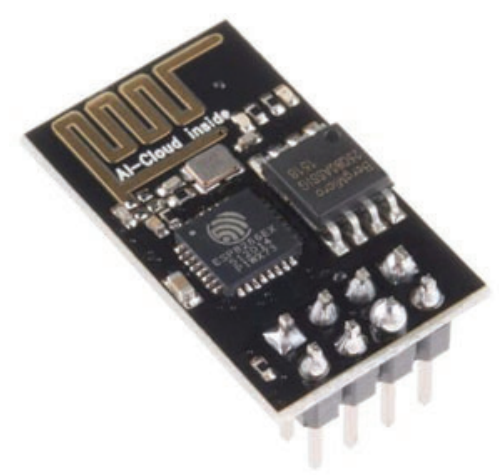

Figure 7: ESP8266 module

Mode 1: Station mode. In this mode, the module allows the microcontroller to connect to WiFi networks (as a client).

Mode 2: Access point mode. In this mode, the module acts as the WiFi host and supplies Internet access to the devices (as a host).

Technical features of an ESP8266 module as follows:

- Memory: The module consists of a $64 \mathrm{~Kb}$ RAM used for instruction and $96 \mathrm{~Kb}$ used for data and also has a flash memory of $1 \mathrm{MB}$.

- WiFi: The module has a TCP/IP protocol integrated and also uses an $802.11 \mathrm{~b} / \mathrm{g} / \mathrm{h}$, which is a single band protocol that can deliver $2.4 \mathrm{GHz}$ speed.

- CPU: The module consists of a 32-bit RISC CPU and a 32-bit application CPU.

\subsection{6 $16 \times 2$ LCD Display}

The LCD module is used for displaying the readings and the status of the design. The pixel size of the LCD screen is 1602 (16 by 2), and the display color is BLUE. In this device, the LCD screen shows the welcome message and also displays the level of concentrations of each gas we are measuring. Fig. 8 depicts the $16 \times 2$ LCD deployed for this project. The command register stores the commands sent to the display, whereas the data displayed are stored in the data register. The data of the intended image are stored in the data register and the instructions in the command register to control the display. The contrast of the display is adjusted by using a potentiometer connected across the VEE pin. The technical specifications are as follows:

- Makes a $5 \times 8$ and $5 \times 10$ dot matrix possible on its surface.

- Supports a low-power operation: 2.7 to $5.5 \mathrm{~V}$.

- Corresponds to the high-speed micro processing unit bus interface at $2 \mathrm{MHz}$.

- Operates at a 5-V logic level.

- Can display 16 characters in two lines.

\subsection{Arduino Integrated Development Environment}

Fig. 9 shows the Arduino Integrated Development Environment (IDE). IDE is an opensource application. In the IDE, programs are called a sketch, and its extension, when saved, is an .ino file. The programming language used in writing a sketch is $\mathrm{C}$ language. The Arduino IDE consists of an editor for code writing, a text console, toolbars that contains a button with functions and a series of menus, a message area for displaying status and errors, and a series of 
menus. The toolbar button allows for the saving of sketch, code verification, serial monitoring, and program upload. When a sketch is uploaded, the Arduino's bootstrap is utilized, which is a stacked program to the board, supporting the program to be loaded without any additional hardware.

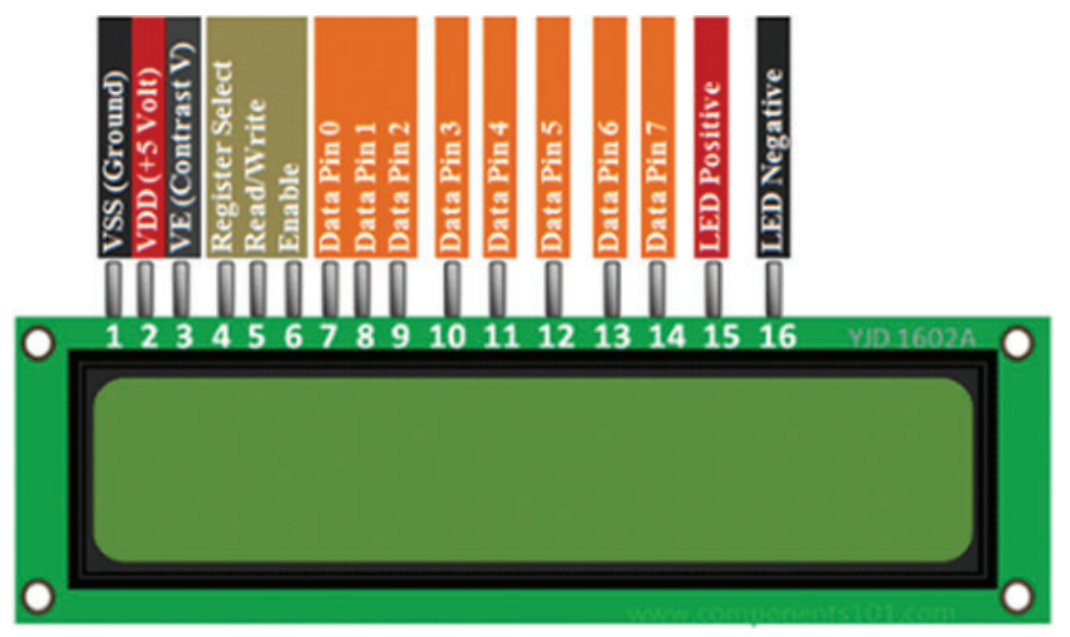

Figure 8: LCD module

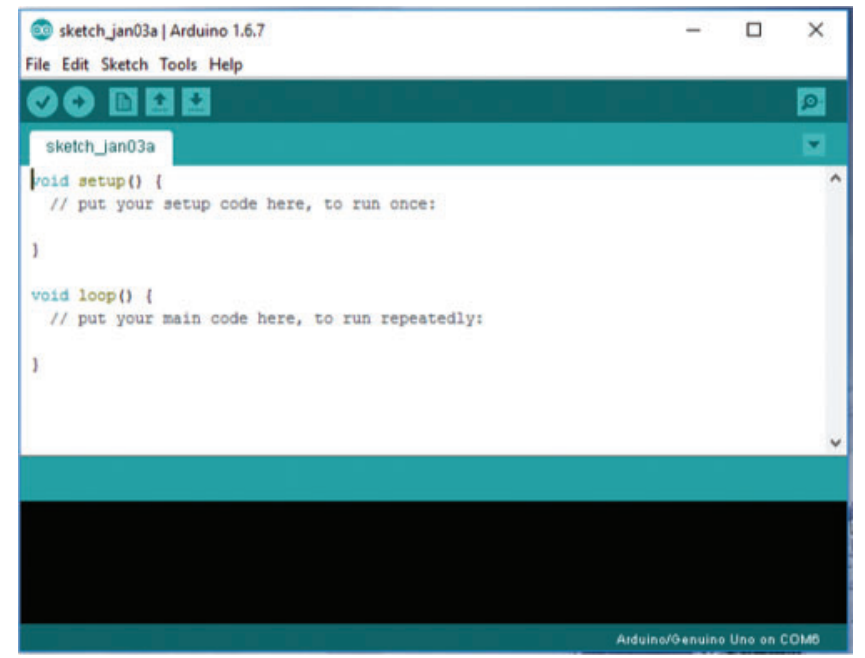

Figure 9: Arduino integrated development environment (IDE)

\subsubsection{ThingSpeak IoT Platform}

ThingSpeak is an open-source IoT-based API used to retrieve and store data from IoT-based devices through the HTTP protocol over the Internet or via a Local Area Network. ThingSpeak allows users to analyze and store in clouds without the need of configuring servers. This platform also allows creating event-based status alerts that trigger based on the incoming data from connected devices. Thingspeak is an IoT platform supported by the numerical computing software 
known as Matlab, which allows its users to analyze and visualize data uploaded to it, thereby linking IoT devices, such as temperature, pressure, and level sensors or microcontrollers Node MCU, Arduino, Raspberry Pi, and others. The main element that worked when using Thingspeak is the channels containing data field, location field, and status field, which can be written to, processed, and viewed with Matlab code. The processed data could also be used to trigger actions, such as sending tweets and other forms of alert.

\section{Implementation}

This section contains a detailed description of the implementation and testing of an IoT-based air quality monitoring system. The working operation of the project at all levels is explained in detail. For a system to meet the stated requirements, each subunit's proper functioning that makes up the system is required because a fault in one unit can affect the functionality of the entire system. Therefore, each subunit was tested to eliminate errors in the implementation and design that may occur during the circuit implementation.

\subsection{Implementation Using Fritzing}

Fritzing is an open-source initiative that is used for schematic and printed circuit board (PCB) hardware simulation and design. The system circuitry framework, schematic, and PCB layout were executed using the Fritzing software. Fritzing was the preferred option because of its extensive library, which contains all the components used in the system. The simulation was conducted to ensure that the system operation was working properly before the commencement of the system development. Fig. 10 shows the breadboard pin connection view.

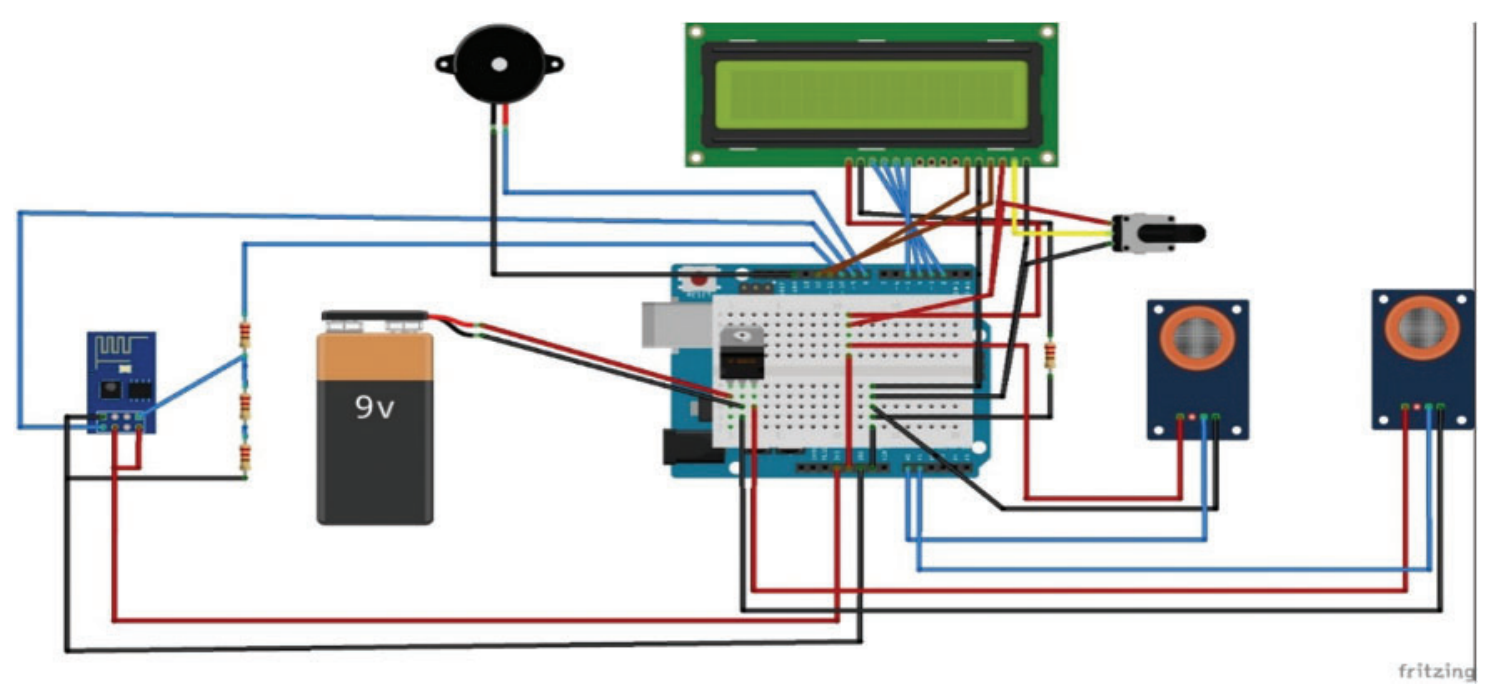

Figure 10: Pin connection of the entire system (modeled with Fritzing)

\subsection{Hardware Implementation}

This section focuses on the actual hardware implementation of a low-cost air quality monitoring system using the Arduino microcontroller. The design's hardware component consists of the ESP8266 WiFi module, LCD module, and the MQ135 and MQ-7 sensors. 


\subsubsection{ESP8266 WiFi Module}

The ESP8266 WiFi module is the communication module and provides the necessary communication protocols to enable the IoT implementation as shown in Fig. 11. The module comprises eight pins, which are Ground, Tx, GPIO-2, GPIO-0, CH_PD, Reset, Rx, and VCC. The CH_PD and the VCC pins are connected to the $3 \mathrm{~V} 3$ pin of the Arduino nano. Then, the transmission pin is connected to digital pin 10 and the receiver pin to digital pin 11 of the MCU. For this implementation, the module is set to operate as a client. Therefore, the module is set to station mode and finally connected to a WiFi network. The module was programmed to retrieve concentration readings of the sensors and update it on the ThingSpeak platform. The Tx pin is connected to pin 10 of the Arduino, and the $\mathrm{Rx}$ pin is connected to pin 9 of the Arduino.

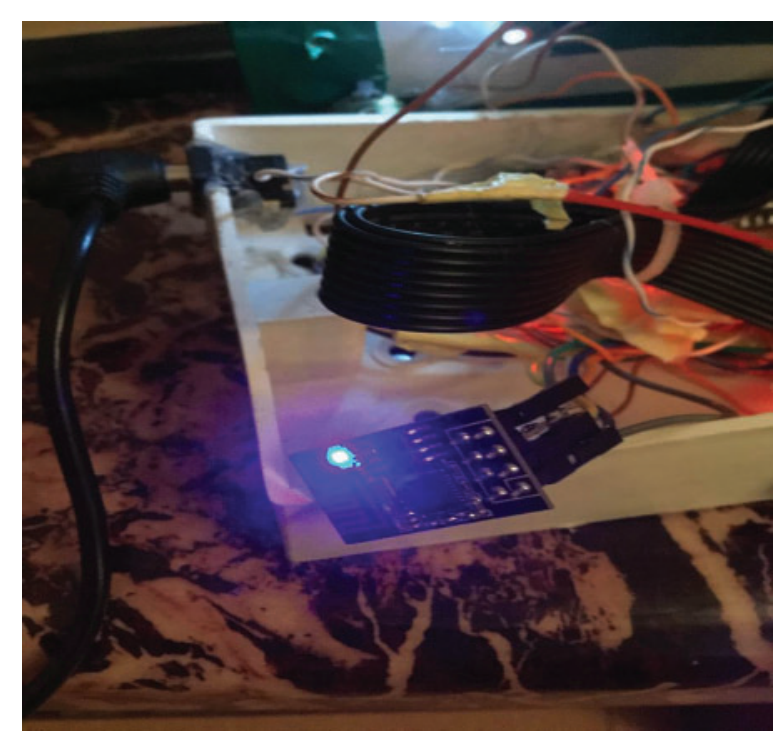

Figure 11: Implemented WiFi module

\subsubsection{LCD Module}

The LCD module consists of 16 pins that control its operation. This module has two operating modes, which are the 8-bit and 4-bit modes. In the former, all the data line pins are used for transmitting and receiving data, whereas in the latter, only pins 4-7 of the data line pins are used. The LCD module was implemented in the 4-bit mode to reduce the microcontroller's number of connections. The LCD module was programmed to display the concentration values from the sensors and the WiFi module's connection status. The LCD is connected to the microcontroller through a connector cable. The register select connection is connected to digital pin 9. The potentiometer's wiper is connected to Vo, and the potentiometer was set to vary the contrast of the display. The Enable (E) pin is connected to digital pin 9 on the Uno board. Then, the 4-bit data pins (D4 through D7) are connected to digital pins 7-5 and finally digital pin 4. The read/write is grounded. Fig. 12 shows the fully implemented LCD module.

\subsubsection{MQ135 and MQ-7 Sensors}

The first step in operating the MQ135 and MQ-7 sensors is to calibrate the sensors for fresh air and create a conversion for the voltage readings obtained from the sensor to standard PPM 
values. Fig. 13 depicts the fully connected settings, and the steps in configuring the sensors are as follows:

- Step 1: The VCC of the air quality sensors is connected to the $5 \mathrm{~V}$ pin of the MCU, and the ground pin is connected to the ground of the MCU. Then, the AOUT pin is connected to the Analog pins of the MCU. The microcontroller reads the gas module with the use of analog to digital converter pins.

- Step 2: The sensors are first connected for a preheating period to find the value of fresh.

- Step 3: The microcontroller was then programmed to check for the threshold value where the gas concentration should exceed. Then, the buzzer alarm and the LED would go off, notifying nearby people that the air is unsafe to breathe.

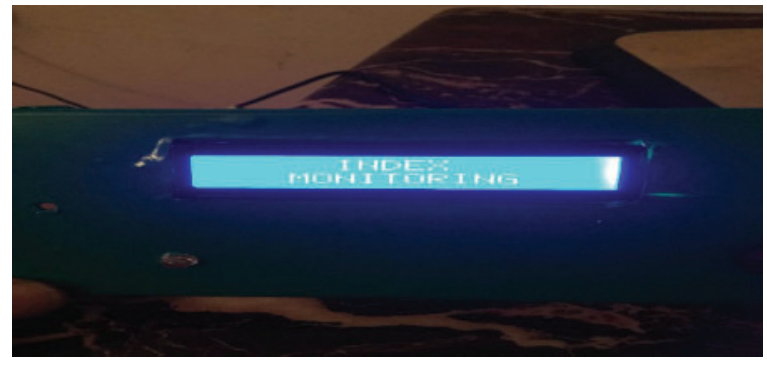

Figure 12: Implemented LCD module

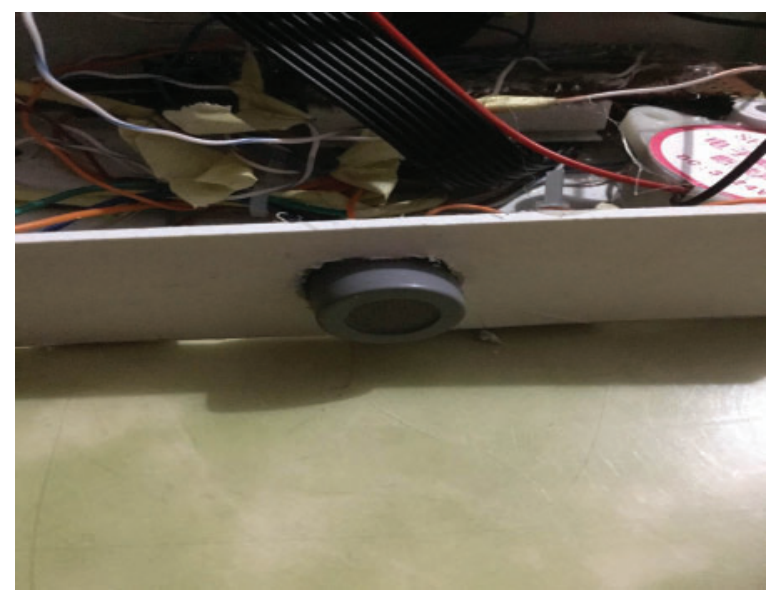

Figure 13: Diagram of the implemented air quality sensor

\subsection{Software Implementation}

The software implementation of this project was conducted using the Arduino IDE and ThingSpeak platform. The former provides an enabling environment for the microprocessor configuration, whereas the latter is responsible for the real-time implementation of the IoT of the project.

\subsubsection{Arduino IDE Programming}

The Arduino IDE is used for coding, setting up, and testing the components. The version used is version 1.8.13. The steps for setting up the software are listed as follows. 
(1) Download and install the Arduino IDE.

(2) Install the board on the IDE, which is conducted by accessing the tools menu and setting the board option to nano.

(3) Load the libraries into the IDE, from the library function in the sketch tab; the needed libraries are loaded and installed.

(4) Writ the code on the coding area, which is then uploaded to the board through the upload function.

\subsubsection{ThingSpeak Platform}

The ThingSpeak platform is for displaying the value of the concentration on its channels as shown in Figs. 14 and 15. Two channels were created to publish the data, where one channel is for the air quality concentrations and the other is for carbon monoxide concentration. The channel is set as a continuous line graph that updates every $30 \mathrm{~s}$. The graph is constructed as concentration against time.

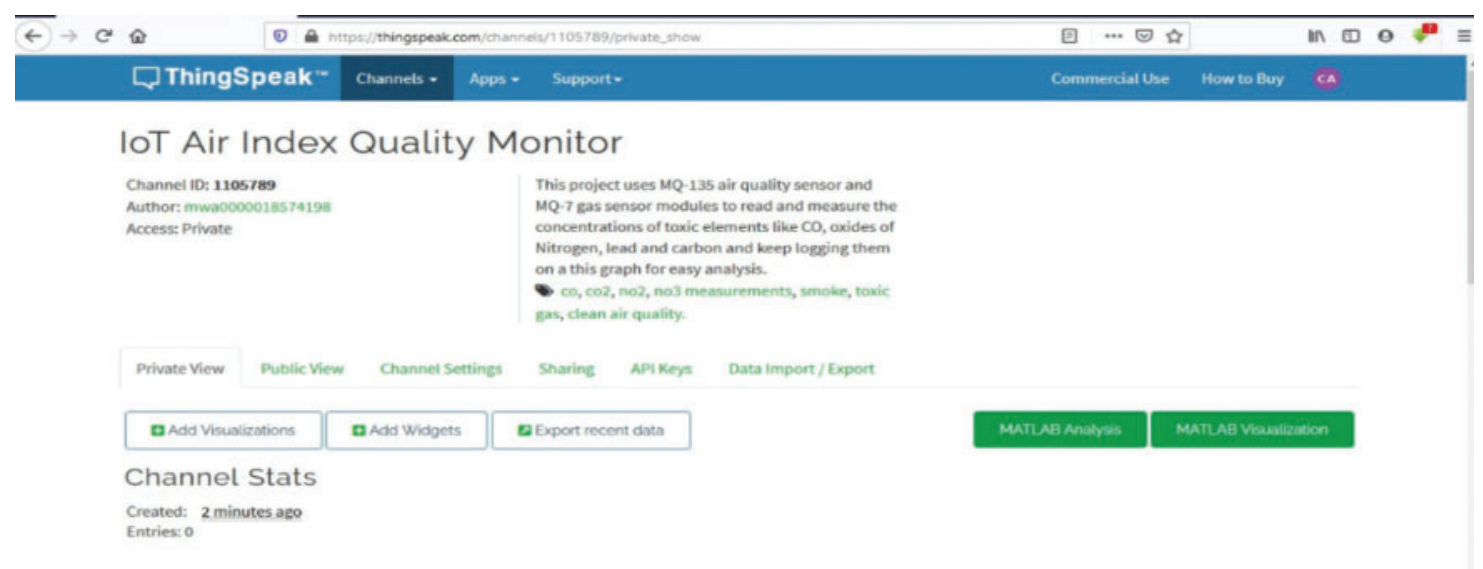

Figure 14: Setting up the ThingSpeak channels

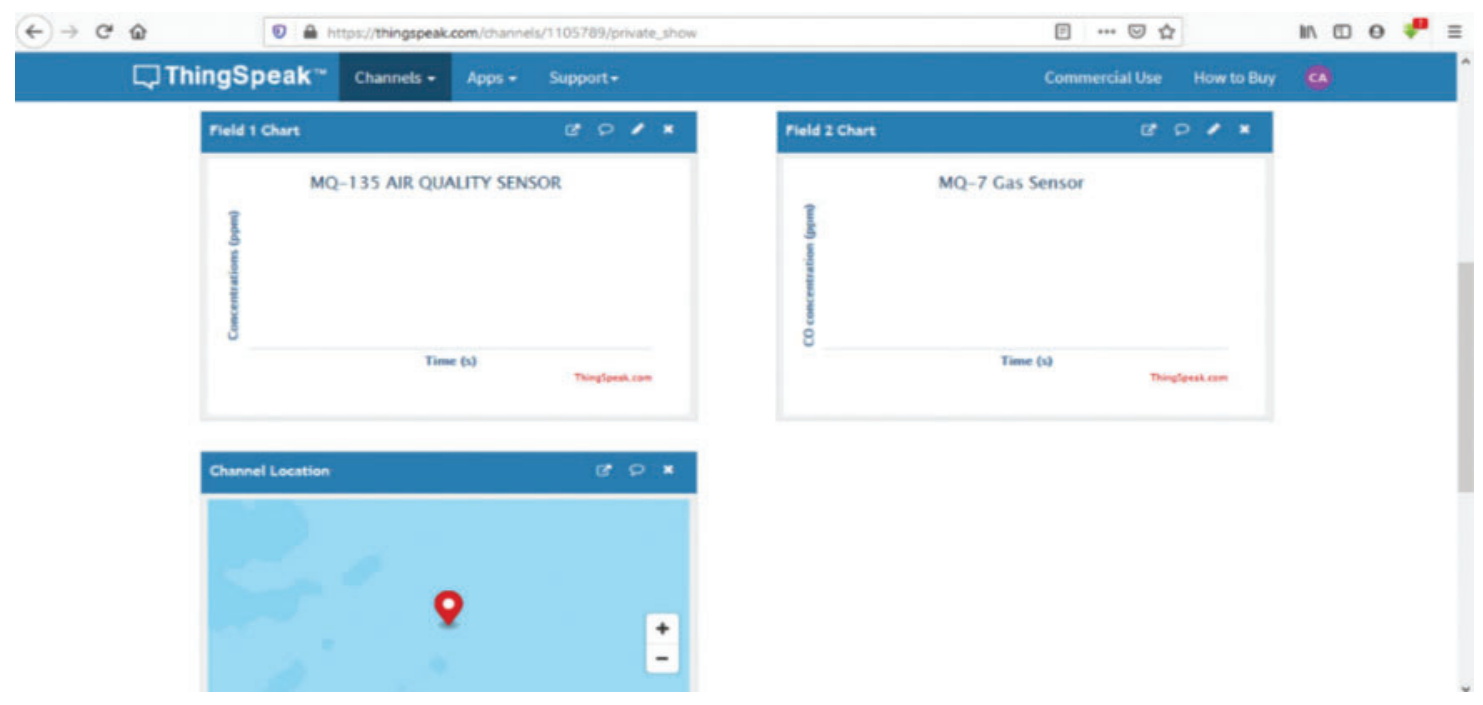

Figure 15: ThingSpeak channels 


\section{Results and Discussions}

Several different tests were conducted to ensure that the proposed system meets the requirements. The system components were tested individually to ensure continuity and their power ratings through the use of a multi-meter. The test was done before integrating into the system to ensure their functionality. This type of testing is referred to as unit testing. Afterward, sub-unit testing was implemented. Each sub-unit was tested to ensure its functionality before integrating it into the proposed system. In this stage, the sensors and modules were tested to ensure that they interacted with the microcontroller. The components were placed correctly to determine the current and voltage consumption. Moreover, system testing involving the entire air monitoring testing was performed. In this stage, the proposed system was tested completely with all the sub-units and components connected, as shown in the diagrams. During the testing, the system was supplied with power, and the system worked perfectly. Then, the system was packaged and enclosed in a green physical case. To ensure that the design satisfies the design goals and objectives, an acceptance test was implemented. In this stage, the code for the system program was debugged and compiled to ensure that all errors were fixed. This stage also ensures that the program codes meet all the system functions.

The proposed system was connected to a power supply and tested to ensure that the system meets the requirement. The MCU reads the sensor's values and transmits them through the WiFi module when connected to a WiFi network. The transmitted data are observed on the ThingSpeak platform for $30 \mathrm{~s}$. When the concentrations become harmful, the system initiates a buzzer and blinks the LED. The data are also displayed on the LCD, which also displays the functionality of the other components. Fig. 16 shows that the MQ-135 sensor registered the quality of air in a particular area. Evidently, the quality of air is not good. When the AQI is good, the AQI category starts at 0 and ends at 50. This category is symbolized in green color. Air quality is deemed to be good, and air pollution presents nearly no effect or hazard. However, AQI is considered moderate. That is, the AQI category starts at 51 and ends at 100. This category is symbolized with a yellow color. Air quality in this category is deemed tolerable. However, harmful pollutants still exist, which will likely affect the health of a limited set of individuals. For instance, individuals sensitive to ozone encounter respiratory defects. This case is another class when the AQI is considered unhealthy for sensitive groups: This AQI category starts at 101 and ends at 150, which is symbolized in orange color. This category is not likely to affect the general health of the public. Moreover, people with lung diseases, older patients, and children are at higher risk from exposure to ozone. Furthermore, those with heart and lung diseases, older patients, and children are at a higher risk of particles' exposure in the air. The AQI is termed unhealthy at 151 and ends at 200. This category is symbolized in red color. In this category, individuals start noticing serious health challenges. Older adults and children may encounter health challenges, particularly individuals with lung or heart diseases. This category is symbolized in purple color. This AQI category triggers health alerts stating that every individual may experience severe health impacts. Among them, the most dangerous is when the AQI is considered hazardous. This case occurs when the AQI is greater than 300. This category is symbolized in a maroon color. Health warnings are indicated, and awareness is created as air pollution at this level has become life-threatening.

The $\mathrm{CO}$ is an odorless and colorless gas developed during incomplete incineration of fuels, such as coal, wood, or natural gas. $\mathrm{CO}$ is a very toxic gas that is fatal when inhaled in large quantities. Fig. 17 highlights the performance of the air quality system using the $\mathrm{CO}$ gas. The biggest sources of $\mathrm{CO}$ are automobiles and machinery that combust fossil fuels. $\mathrm{CO}$ cannot be identified by the human senses considering that this gas has no odor, taste, or color. This notion 
means that harmful concentrations can develop indoors, and humans do not have a way to detect it. When humans are exposed to $\mathrm{CO}$ gas, the $\mathrm{CO}$ gets into the bloodstream, where it displaces and reduces the amount of oxygen that is transported to vital organs, such as the brain and heart. The study proposed a similar project, that is, the system "N-SMARTS." However, different from the fact that the proposed system consists of more than one sensor, the system collected data on air pollutants by interfacing GPS-enabled mobile phones with sensors. The system provided a simple and accessible system for detecting air pollutants using low-cost sensors.

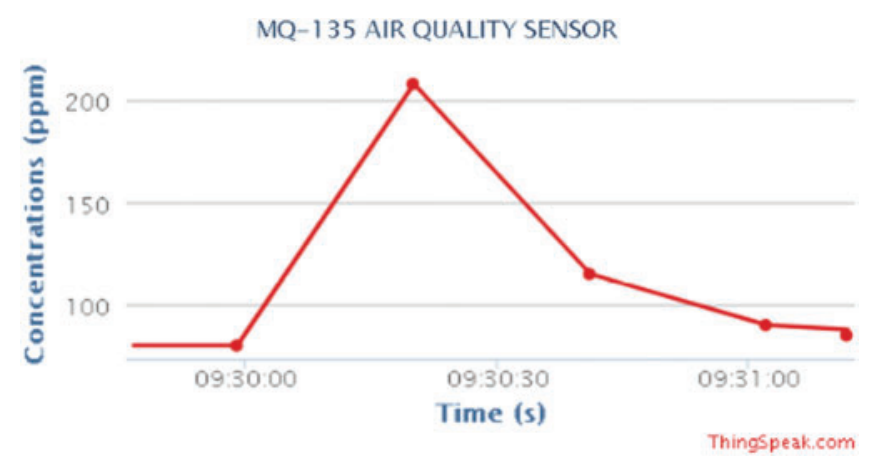

Figure 16: Results of the MQ 135 channel

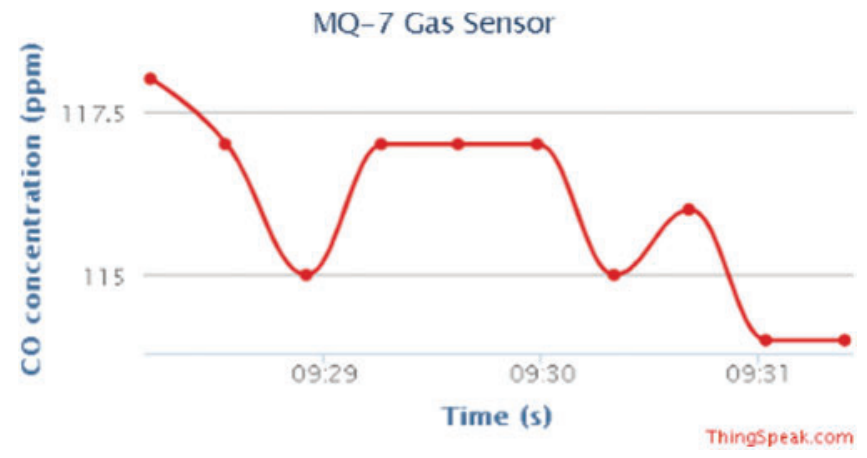

Figure 17: CO level consideration

\section{Conclusion}

This work successfully implemented a design of a low-cost air quality monitoring system using Arduino and ThingSpeak, showing that an air quality system can be implemented using a lowcost technology, Arduino and ThingSpeak. The executed project was tested using various testing categories, namely, unit, sub-unit, system, acceptance, and program testing. The system performs credibly well in all testing parameters. With this system, a mobile app that can be easily deployed to monitor the air quality across the spectrum can be developed, thereby improving the health of the populace.

Funding Statement: This work was supported by SUT Research and Development Funds and by Thailand Science Research and Innovation (TSRI). In addition, this work supported by the Taif 
University Researchers Supporting Project number (TURSP-2020/77), Taif University, Taif, Saudi Arabia.

Conflicts of Interest: The authors declare that they have no conflicts of interest to report regarding the present study.

\section{References}

[1] S. T. Odonkor and T. Mahami, "Knowledge, attitudes, and perceptions of air pollution in Accra, Ghana: A critical survey," Journal of Environmental and Public Health, vol. 2020, no. 9, pp. 1-8, 2020.

[2] P. Mehndiratta, A. Jain, S. Srivastava and J. E. Gupta, "Environmental pollution and nanotechnology," Environment and Pollution, vol. 2, no. 2, pp. 49-54, 2013.

[3] Y. Huang, Q. Zhao, Q. Zhou and I. A. Jiang, "Air quality forecast monitoring and its impact on brain health based on big data and the internet of things," IEEE Access, vol. 6, no. 5, pp. 78678-78688, 2018.

[4] K. Bianchini, R. Alvo, D. C.Tozer and M. L. Mallory, "The legacy of regional industrial activity: Is loon productivity still negatively affected by acid rain," Biological Conservation, vol. 255, no. 3, pp. 108977-108997, 2021.

[5] D. Rickerby, M. J. S. Morrison and T. O. Aly, "Materials, nanotechnology and the environment: A European perspective," Science and Technology of Advanced Materials, vol. 8, no. 2, pp. 19-30, 2007.

[6] D. E. Williams, G. S. Henshaw, M. Bart, G. Laing, J. Wagner et al., "Validation of low-cost ozone measurement instruments suitable for use in an air-quality monitoring network," Measurement Science and Technology, vol. 24, no. 6, pp. 65803-65823, 2013.

[7] A. C. Lewis, J. D. Lee, P. M. Edwards, M. D. Shaw, M. J. Evans et al., "Evaluating the performance of low cost chemical sensors for air pollution research," Faraday Discussions, vol. 189, no. 5, pp. 85-103, 2016.

[8] P. Arroyo, F. Meléndez, J. Suárez, J. Herrero, S. Rodríguez et al., "Electronic nose with digital gas sensors connected via bluetooth to a smartphone for air quality measurements," Sensors, vol. 20, no. 3, pp. 786-806, 2020.

[9] B. Bishoi, A. Prakash and V. J. A. Jain, "A comparative study of air quality index based on factor analysis and US-EPA methods for an urban environment," Aerosol and Air Quality Research, vol. 9, no. 1, pp. 1-17, 2009.

[10] M. Ansah, R. Sowah, J. Melià-Seguí, F. Katsriku, X. Vilajosana et al., "Characterising foliage influence on LoRaWAN pathloss in a tropical vegetative environment," IET Wireless Sensor Systems, vol. 10, no. 5, pp. 198-207, 2020.

[11] S. Kaivonen and E. Ngai, "Real-time air pollution monitoring with sensors on city bus," Digital Communications and Networks, vol. 6, no. 1, pp. 23, 2020.

[12] V. Vinod, V. Mekala, S. Abinaya, A. Srinivas and S. Arun, "A customizable cartographic air pollution monitoring system," International Journal of Scientific and Technology, vol. 9, no. 4, pp. 1675-1678, 2020.

[13] R. Dhanusha and S. Rathi, "A survey on air pollution monitoring using internet of things," International Journal of Scientific Research in Science, Engineering and Technology, vol. 7, no. 3, pp. 350-355, 2020.

[14] P. Souza, A. Anjomshoaa, F. Duarte, R. Kahn and C. Ratti, "Air quality monitoring using mobile lowcost sensors mounted on trash-trucks: Methods development and lessons learned," Sustainable Cities and Society, vol. 60, no. 3, pp. 102239- 102248, 2020.

[15] N. Motlagh, E. Lagerspetz, P. Nurmi, X. Li, S. Varjonen et al., "Toward massive scale air quality monitoring," IEEE Communications Magazine, vol. 58, no. 2, pp. 54-59, 2020.

[16] K. Zheng, S. Zhao, Z. Yang, X. Xiong and W. Xiang, "Design and implementation of LPWA-based air quality monitoring system,” IEEE Access, vol. 4, no. 2, pp. 3238-3245, 2016. 
[17] H. Fan, C. Zhao and Y. Yang, "A comprehensive analysis of the spatio-temporal variation of urban air pollution in China during 2014-2018," Atmospheric Environment, vol. 220, no. 5, pp. 117066-117086, 2020.

[18] V. Masson, A. Lemonsu, J. Hidalgo and J. Voogt, "Urban climates and climate change," Annual Review of Environment and Resources, vol. 45, no. 3, pp. 411-444, 2020.

[19] O. A. Postolache, J. D. Pereira and P. Girao, "Smart sensors network for air quality monitoring applications," IEEE Transactions on Instrumentation and Measurement, vol. 58, no. 9, pp. 3253-3262, 2009.

[20] N. Kularatna and B. H. Sudantha, "An environmental air pollution monitoring system based on the IEEE, 1451 standard for low cost requirements," IEEE Sensors Journal, vol. 8, no. 4, pp. 415-422, 2008.

[21] M. Taştan and A. S. Gökozan, "Real-time monitoring of indoor air quality with internet of thingsbased E-nose," Applied Sciences, vol. 9, no. 16, pp. 3435-3455, 2019. 